\title{
A USER-CENTERED DESIGN OF A MAJOR AND MINOR INTRON ANNOTATION DATABASE (MMIAD)
}

\author{
Ruby ElKharboutly ${ }^{1}$, Kyle Gorman ${ }^{1}$, William Eccles ${ }^{1}$ and Sahar Al Seesi ${ }^{2}$ \\ ${ }^{I}$ Quinnipiac University, CT, USA \\ ${ }^{2}$ Southern Connecticut State University, CT, USA
}

\begin{abstract}
Biologists and bioinformaticians heavily rely on data portals and repositories accessible through web application. While they mostly agree that the data is valuable, they find the interfaces hard to use and non-intuitive. In this paper we present a user-centered design of a database for classification and annotation for major and minor introns in various species. Our design is based on surveying and interviewing minor intron researchers and comparing the features of existing databases. In addition to its ease of use, the proposed database, Major and Minor Intron Annotation Database (MMIAD) offers high flexibility in querying and downloading subsets of information that interest the user in multiple commonly used file formats.
\end{abstract}

\section{KEYWORDS}

Bioinformatics, Introns, User-Centered Development

\section{INTRODUCTION}

Biologists and bioinformaticians heavily rely on web-based systems to retrieve data. Their efforts suffer due to poor usability of system interfaces (Javahery, Seffah, \& Radhakrishnan, 2004; Holtzblatt, Wendell, \& Wood, 2004). Such systems are usually developed from a programmer perspective to provide specific functionality giving little to no consideration to usability (Pavelin, Cham, de Matos, Brooksbank, \& Cameron, 2012). These applications fail to meet users' needs and expectations requiring significant effort and time. One example is UCSC Table Browser (D, et al., 2004). The user interface for this tool is overly crowded and hard to read. It uses outdated UI controls and is built using CGI. Many of the input controls are non-initiative and require extra effort to understand. Finally, the Help tool is accessible through extra navigation which make it difficult to use.

This paper illustrates how software engineering and usability practices are used to elicit the requirements and develop the design of Minor and Major Intron Annotation Database (MMAID) by following a User-Centered Development (UCD) approach (Mao, Vredenburg, Smith, \& Carey, 2005). Most bioinformatics tools ignore usability requirements and are hardly comparable to software designed for other domains (Rose, 51-60; Pavelin, Cham, de Matos, Brooksbank, \& Cameron, 2012; Javahery, Seffah, \& Radhakrishnan, 2004). There has been considerable effort that addresses the usability needs of biologists and bioinformaticians. Bolcini et. al. (Bolchini, Finkelstein, Perrone, \& Nagl, 2009) discuss usability issues in a number of web-based search engines. They suggest recommendations for improvement for these websites. Javahery et. al. (Javahery, Seffah, \& Radhakrishnan, 2004) describe their work in applying UCD while developing an integrated web-based interface to a popular bioinformatics portal such that access to web resources is optimized. Pavelin (Pavelin, Cham, de Matos, Brooksbank, \& Cameron, 2012) worked on evaluating a number of projects and adopted a UCD approach to produce designs that satisfy usability requirements. de Matos et al. present a case study on how they applied UCD when creating a website that provides enzyme-related data.

As a result of the applying a UCD approach, we are proposing MMAID, user-centered, intuitive, task-based, and most importantly easy to update database that annotates major and minor introns in different species. MMIAD will support multiple genome and transcriptome versions for each species in the database. It will offer high flexibility in querying and downloading subsets of information that interest the user in multiple commonly used file formats. 


\section{BACKGROUND AND RELATED WORK}

Most expressed genes go through the process of intron splicing. The cell machinery responsible for splicing identifies the introns by specific patterns at their boundaries (splice junctions). There are two sets of proteins that perform this job: the major spliceosome responsible for splicing the majority of introns (U2 or major introns) and the minor spliceosome responsible for splicing the rest of the introns (U12 or minor introns). (Sharp \& Burge, 1997). There is a lot of interesting biological research that focuses on understanding the differences between these two splicing machineries and their effect on different biological phenomena (Baumgartner, et al., 2018; Madan, et al., 2015), and this research depends on existing annotations for minor introns. All the research that addresses minor introns uses the annotations from two databases that were developed in 2006, SpliceRack (Sheth, et al., 2006) and U12DB (Alioto, 2006). Both databases were created based on the genome and transcriptome versions at their time of creation and were not updated. As a result, numerous preprocessing steps are needed when using this outdated data. In this work, we aim to develop a new user-friendly and easily maintainable database that annotates introns and classifies them into major and minor introns.

The foundation work for classifying introns as either major or minor was laid out by Sheth et. al (Sheth, et al., 2006) using position weight matrices (PWM). They built a resource where the PWMs and annotated introns can be queried and downloaded for five species. Alioto et. al. (Alioto, 2006) built on this work and created a web-based repository that contains U12 introns for 24 species. Their repository included more query features and additional information, One of the main problems with both resources is that they were never updated with new intron coordinates, intron sequences, or gene IDs and links. Both resources were published in 2006, and the genome and transcriptome annotations of different species have been updated multiple times since then.

Very recently, Othlof et. al. (Olthof, Hyatt , \& Kanadia, 2019) created the minor intron database (MIDB), with updated intron and gene information for human and mouse using PWMs. In addition to including annotations for only two species, the search feature of MIDB is restricted to searching by gene name or Ensembl ID. Moyer et al (Moyer, Larue, Hershberger, Roy, \& Padgett, 2020) created The Intron Annotation and Orthology Database (IAOD). They use PWMs coupled with a support vector machine to classify the introns. Their database includes 24 species and they offer a wide range of search capabilities.

\section{METHODS}

Although most bioinformatics tools are built to run on Linux systems and are accessible through the command line, due to their high computational complexity, the end users of these tools are biologists with very limited experience in using Linux and command line. On the other hand, some of the available databases, like U12DB and SpliceRack, do not allow bioinformaticians to interface with them through APIs. That calls for incorporating UCD in the development process of these tools and databases. An evaluation of the existing U12 databases, was performed to identify missing features and usability short comings. We also collected requirements from target users through interviews and a survey. Our target users included eleven biologists and bioinformatics researchers.

\subsection{Survey Design}

The survey was designed such that it evaluates the effectiveness of commonly used U12 database, U12DB, in functionally providing data to the users as well as the usability aspects of the website. Usability aspects included ease of use and clarity. The survey was distributed via email to participants. The following is a list of questions included in the survey.

1. What is your field of study?

2. What is your main purpose in using the U12DB site?

3. Was the information easy to find? $(\mathrm{y} / \mathrm{n})$

4. Was the information clearly presented? $(\mathrm{y} / \mathrm{n})$

5. Were you able to find what you were looking for? $(\mathrm{y} / \mathrm{n})$ 
6. When using the U12BD site or similar websites, what would you say is the most important feature you look for?

7. Which of these output formats would you make use of?

- Intron Gff

- Intron Fasta

- Donor

- GTF

- BED

- User Defined Tabular File Format

- Other

8. What other features would you add to the website?

\subsection{Interview Questions}

We performed in-depth interviews with two bioinformatics scientists and one biologist to further understand the user requirements and usability challenges. We asked the interviewees to inspect each aspect of the U12DB UI such as query text and query options, tabular output, single intron output, and help options. We also asked the interviewees if the purpose of each feature is clear, whether it is intuitive and if it is correctly placed in the UI. Moreover, we solicited users' suggestions for changes in the UI and/or functionality.

\subsection{Similar Websites Comparison}

We performed a detailed comparison between the U12 database, and two other databases, namely MIDB and IAOD, that were very recently published. Despite the fact that SpliceRack is a valuable resource for intron splice junction PWMs, we found it to be less comprehensive in terms of features and usability; therefore, it was excluded from the comparison. The details of the comparison are presented in section 3.3.

\section{RESULTS}

\subsection{Survey Results}

The survey was emailed to around thirty biologists and bioinformaticians. Only eight responses where received from one biologist and seven bioinformaticians.

A summary of the resulted of questions 3-6 is presented in Table 1, and the statistics for question 7 responses are presented in Figure 1.

Table 1. Results of Questions 3,4 and 5

\begin{tabular}{|l|l|l|}
\hline Question & Yes & No \\
\hline Was the information easy to find? & $75 \%$ & $25 \%$ \\
\hline Was the information clearly presented? & $75 \%$ & $25 \%$ \\
\hline Where you able to find what you are looking for? & $12.5 \%$ & $87.5 \%$ \\
\hline
\end{tabular}

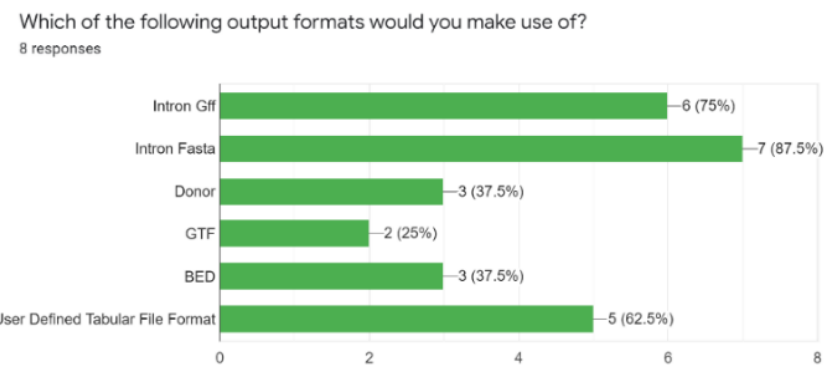

Figure 1. Responses to Survey Question 7 
In summary, about a quarter of the population found the website interface confusing and the information presented unclear. A significant number of participants found it hard to access specific website features due to problems in navigation and confusing help menu. All participants agreed that the U12DB is lacking in the following two aspects:

- Updated intron information according to the latest genome and transcriptome versions

- Easier extraction of the sequence of the gene harboring the U12 intron

\subsection{Interviews Results}

The in-depth interviews were very useful in identifying missing features and usability challenges. The following features were found missing from the U12DB website:

- Allow the user to query by genome locus and query for all the introns in a certain chromosome.

- Allow the user to search by sequence

- Include the score, location and subtype of the intron in the output page

- Export results to comma separated format

- Allow the user to obtain the search results via email

- Make the interface more user friendly and the query criteria organized in a better way

- Generate output with user defined fields

- Change the color scheme to less flashy colors

- Include the complete set of U2 introns

- Enable the user to predict U12 introns for genomes other than the ones in the database

- Offer a more user-friendly website help feature

\subsection{Comparison Results}

We compared U12DB, MIDB, IAOD, and our proposed MMIAD databases in terms of the species included in each database, the different criteria by which the user can query the database, the data included in the output and the supported output formats. We also included in the comparison other features such as search response time, interface methods, and the prediction algorithms. Table 2 details the results of this comparison.

The comparison shows that among the three databases, IAOD has the most flexible query options and the most informative output. U12DB has the advantage of making the database accessible through a SQLDump. This gives the users the ability to download the data and query it by embedding SQL queries in their code. Each of the three databases have a number of downloadable file formats, but both MIDB and IAOD have this feature available only for the whole dataset; meaning that the user cannot download a subset of the data based on a search query. The search feature results are embedded in an HTML page. U12DB does not create downloadable files for search results, but it displays the results in the browser as unformatted text that can be saved to a file.

In addition to the comparison between the three databases, Table 2 also has a column that describes our proposed MMIAD features. These features were selected based on this comparison and the results of the conducted survey and interviews. One of the major advantages of MMIAD over the existing databases is that the user will be able to choose the genome and transcriptome versions. MMIAD will include annotations for different genome and transcriptome versions for each species. None of the other databases offer this feature except for IAOD, which supports only two human genome versions and only one version for the rest of the species. This is crucial because it eliminates the need to convert coordinates or gene IDs if the user is using a different genome or transcriptome version in their research. MMIAD will contain both major and minor introns. Therefore, the user does not need to download the major introns from another source, if needed in their research.

Additionally, MMIAD will provide the user the ability to download a complete transcriptome GTF, needed by most gene expression analysis tools, with extra annotation, showing which exons border minor introns. Another important feature is allowing the users to download the results of their search in multiple commonly used output file formats, such as FASTA, BED, and GTF. In addition to its web interface, MMIAD will also be accessible through REST APIs to enable bioinformaticians to easily integrate MMIAD queries into their analysis pipelines. For web interface users, MMIAD will send a notification email to the user including a link to view and/or download the requested results, which is a useful feature for time intensive queries that usually results in the website timing out in the existing databases. Other features of MMIAD that are related to the user interface are reflected in the web interface figures in Section 3.4. 
Table 2. Comparing the features of the four U12 intron

\begin{tabular}{|c|c|c|c|c|c|}
\hline \multicolumn{2}{|l|}{ Feature } & U12DB & MDB & IAOD & MMAD \\
\hline \multicolumn{2}{|c|}{ Major introns included } & A small subser & No & Yes & Yes \\
\hline \multicolumn{2}{|c|}{ Species } & 20 & 2 & 24 & NA** \\
\hline \multicolumn{2}{|c|}{ Genome versions } & No & No & Only for human & Yes \\
\hline \multicolumn{2}{|c|}{ Transcriptome versions } & No & No & No & \\
\hline \multirow[t]{12}{*}{ Query criteria } & Species & Yes & Yes & Yes & Yes \\
\hline & Ensemb| Gene ID & Yes & Yes & Yes & Yes \\
\hline & Gene name & Yes & Yes & Yes & Yes \\
\hline & Ensembl Transcript ID & No & № & Yes & Yes \\
\hline & Inton Type & Yes & No & Yes & Yes \\
\hline & Inton cluster & Yes & No & No & No \\
\hline & Inton subtype & Yes & No & Yes & Yes \\
\hline & Frame & Yes & No & Yes & Yes \\
\hline & Inton length & No & No & Yes & Yes \\
\hline & Coordinate range & No & No & Yes & Yes \\
\hline & Stand & Yes & No & Yes & Yes \\
\hline & Sequence & No & No & Yes & Yes \\
\hline \multicolumn{2}{|c|}{ Predicfon algorithm } & PWM & PWM & PWM and SWM & $\begin{array}{l}\text { PWM and } \\
\text { probabilistic } \\
\text { grammars }\end{array}$ \\
\hline \multicolumn{2}{|l|}{ Interbace } & $\begin{array}{l}\text { Web in terfaos \& } \\
\text { myscL dump }\end{array}$ & Web interace & Web interáa & $\begin{array}{l}\text { Web interface with } \\
\text { email noffication \& } \\
\text { REST APIs }\end{array}$ \\
\hline \multirow{4}{*}{\multicolumn{2}{|c|}{ Downloada ble formats }} & Intron GFF & Inton xdsx file & U12 FASTA & |Intron BED \\
\hline & & $\begin{array}{l}\text { Intron FASTA } \\
\text { Donor FASTA }\end{array}$ & $\begin{array}{l}\text { Gene } x \text { ssx fle } \\
\text { Inton FASTA }\end{array}$ & $\begin{array}{l}\text { U2 FASTA } \\
\text { U2 } 2 \text { BED }\end{array}$ & $\begin{array}{l}\text { Intron FAST A } \\
\text { Donor exon FASTA }\end{array}$ \\
\hline & & \begin{tabular}{|l|} 
Accoptor FASTA \\
Branch point FASTA
\end{tabular} & $\begin{array}{l}\text { Intron BED } \\
\text { Donor exon BED }\end{array}$ & U12 BED & Acceptor exon FASTA \\
\hline & & & $\begin{array}{l}\text { Acceptor exon BED } \\
\text { Transcript BED }\end{array}$ & & \\
\hline \multicolumn{2}{|l|}{ Notes } & $\begin{array}{l}\text { Not downladable } \\
\text { but can be saved } \\
\text { from browser }\end{array}$ & $\begin{array}{l}\text { Dataset can be } \\
\text { downloaded as a } \\
\text { whole. Search } \\
\text { results are not } \\
\text { downloadable }\end{array}$ & $\begin{array}{l}\text { Dataset can be } \\
\text { downloaded as a } \\
\text { whole. Search result } \\
\text { are not downloasdable }\end{array}$ & $\begin{array}{l}\text { All output iles can be } \\
\text { obtained for data } \\
\text { resulfing fom search } \\
\text { results }\end{array}$ \\
\hline \multirow[t]{37}{*}{ Output felds } & Species & Yes & Yes & Yes & Yes \\
\hline & Common species name & No & No & Yes & Yes \\
\hline & Genome version & No & Yes & Yes & Yes \\
\hline & Ensembl version & No & No & No & Yes \\
\hline & Gene name & Yes & Yes & Yes & Yes \\
\hline & Gene description & Yes & Yes & No & No \\
\hline & Synonyms & No & Yes & No & No \\
\hline & NCBI gene ID & No & Yes & No & Yes \\
\hline & NCBI gene link & No & Yes & No & Yes \\
\hline & Ensembl gene ID & Yes & Yes & Yes & Yes \\
\hline & Ensembl gene link & Yes & Yes & Yes & Yes \\
\hline & UCSC link - gene coordinates & Yes & No & No & Yes \\
\hline & Gene type & No & Yes & No & No \\
\hline & Gene disease associations & No & Yes & No & No \\
\hline & Gene Hman Protein Atas link & No & Yes & No & No \\
\hline & Gene Allen Brain Atlas link & No & Yes & No & No \\
\hline & Gene sequence & Yes & Yes & No & Yes \\
\hline & Stand & Yes (in gif) & Yes & Yes & Yes \\
\hline & Gene coordinates & Yes & Yes & No & Yes \\
\hline & Gene length & No & Yes & No & Yes \\
\hline & Ensembl canonical trascript ID & No & Yes & No & No \\
\hline & Ensembl transcript(s) ID(s) & Yes & Yes & Yes & Yes \\
\hline & Ensembl transcript(s) links & & Yes & & Yes \\
\hline & Internal intron ID & $\begin{array}{l}\text { Yes - links to } \\
\text { intron page }\end{array}$ & No & $\begin{array}{l}\text { Yes - links to } \\
\text { orthologus introns }\end{array}$ & No \\
\hline & Intron cluster ID & $\begin{array}{l}\text { Yes - links to } \\
\text { orthologus introns }\end{array}$ & No & No & No \\
\hline & inton coordinates & Yes & Yes & Yes & Yes \\
\hline & UCSC link - int on coordinates & Yes & Yes & No & Yes \\
\hline & Inton length & No & Yes & Yes & Yes \\
\hline & Inton sequenoe & Yes & Yes & Yes & Yes \\
\hline & Branch point sequence & Yes & Yes & Yes & Yes \\
\hline & Donor splioe site sequence & Yes & Yes & Yes & Yes \\
\hline & Acceptor splice oite sequence & Yes & Yes & Yes & Yes \\
\hline & Soores & Yes & Yes & Yes $=0$ & Yes \\
\hline & Rank (evon number) & No & & Yes & Yes \\
\hline & Inton type (U2/U12) & Yes & Only U1 2 included & Yes & Yes \\
\hline & Inton subtype - dinucleofdes & Yes & Yes & Yes & Yes \\
\hline & Frame & Yes & No & Yyes & No \\
\hline
\end{tabular}

One of the main motivations behind this project is to create an updated and easily maintainable database, to address the problem of U12DB and SpliceRack being outdated. To ensure maintainability, we include in the design of MMIAD an administrator interface through which an automated process of classifying introns into $\mathrm{U} 2$ and U12 and adding a new dataset to the database can be performed. The input to this feature will be a genome FASTA file and a transcriptome GTF file. This feature will make it easy to update our database every time a new genome or transcriptome version is published. This feature will also be available to users through REST APIs allowing the users to classify introns in a genome of their interest (not in our database) into major and minor introns.

* Only U2 introns that are orthologous to U12 introns in other species are included. ** MMIAD has not been populated with intron data yet.***IAOD has one score for the whole introns. No scores given for individual features. 
The three systems have been evaluated in terms of usability guidelines such as intuitive design, ease of learning, efficiency of use, memorability, and error frequency. The three websites have an intuitive design that resembles common repository search tools. U12DB search resembles running an SQL query. The labels such as "alt-spliced" and "type switch" match database fields and are difficult or remember. The help page for this website is located in a different page and requires extra navigation to access. The site takes a long time to respond depending on the search criteria.

MIDB was found intuitive and more user friendly as it provides very limited functionality. The search options are limited to species and search by gene name or Ensemble id. The presented search output for a single intron required significant scrolling and used internal system ids to identify minor introns which is non-initiative for the user. IAOD is the latest database, published in 2020. It has the best usability features compared to older database.

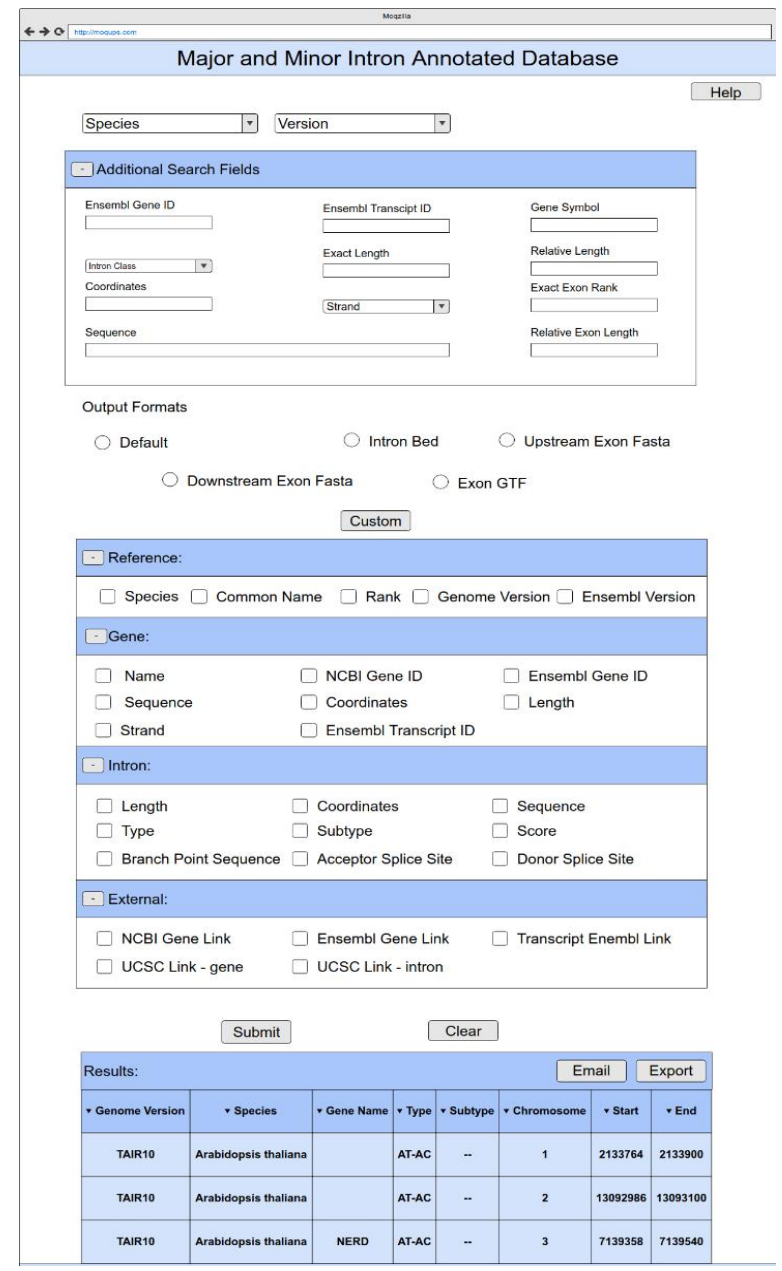

Figure 2. The MMAID homepage through which the user can query the database

\subsection{Website User Interface Design}

Each page of our website was designed with simplicity, clarity, and an intuitive design that is functional as well as user-friendly. We adapt a task-based approach that allows the user to go step-by-step as in the bioanalytical process. The user is provided with the most basic query options in the main search and can optionally choose to expand the search criteria and expose other options. Figure 2 is the landing page. This page provides the main search capability to the user. A simple search by species and genome version is provided. The user is given the option to expand the search criteria by selecting "+ Add Additional Fields". 
The user will also be able to specify the output format of the data. If the user selects the "custom" button under output format, it will display the available output formats. Initially, each of the drop-down menus will be closed and the user will be able to open each in a step-by-step approach. The "Submit" button will run the search and the "Clear" button will clear the page and restore it to the default page. Through the "Email" button, the user can request to receive a notification email when the search is completed. This is useful for time consuming searches, like searching across all species. The "Export" button will bring the user to a popup page that will allow them to export the results to their desired file format. The "Results" table will be visible once the "Submit" button has been selected. By selecting one of the resulting introns, the user will be brought to the "Intron Information" page for that intron which will have all the available information on the intron. Figure 3 shows the intron information page. The user can choose which sections can be displayed on this page, for a more intuitive and cleaner interface

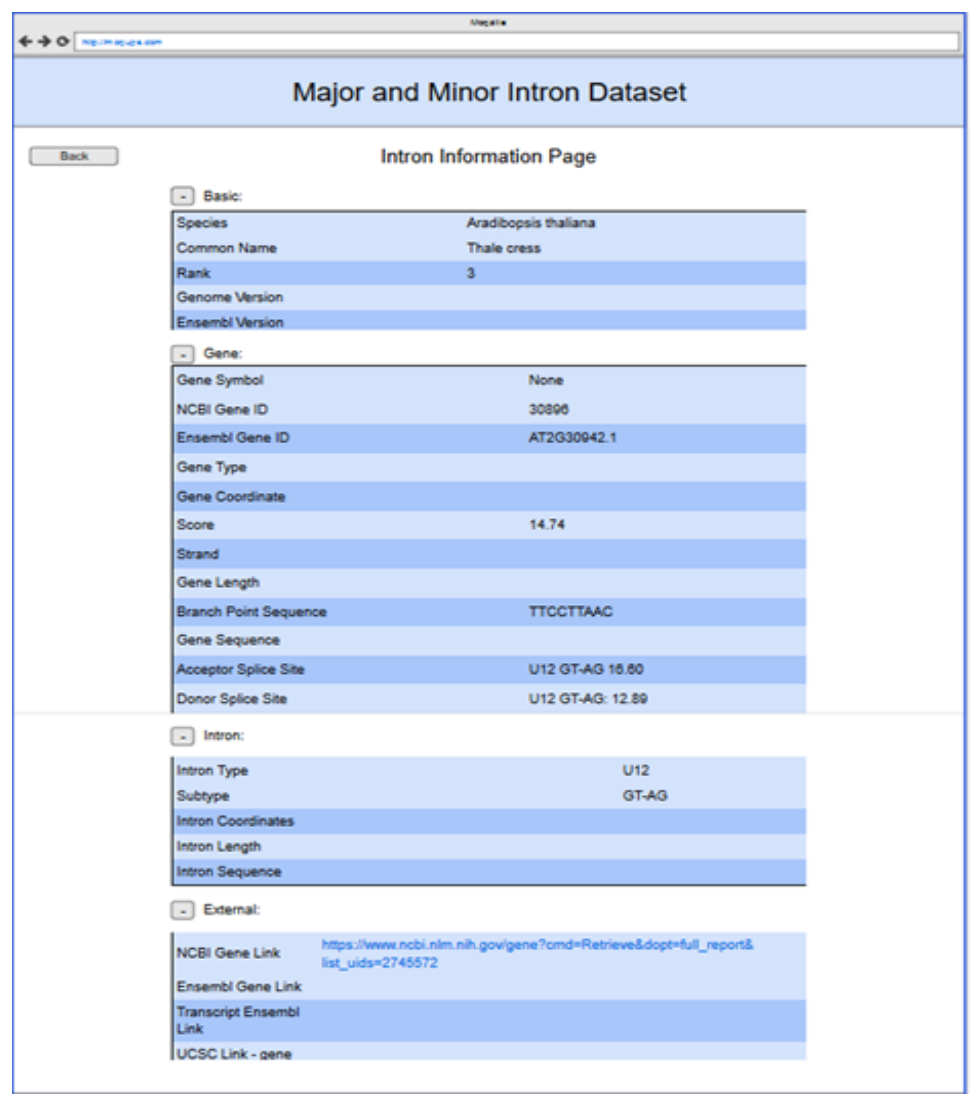

Figure 3. Intron information page

\section{CONCLUSION AND FUTURE WORK}

MMIAD was designed based on a user-centered software development methodology. Our proposed design is simple, intuitive, and user-friendly. MMIAD offers a task-based interface that guides the user in their selection in a step by step process. MMIAD features, output data, and output file formats were selected based on user input acquired from U12DB users through surveys and interviews. We also performed a detailed comparison between the features of U12DB and the two newly created U12 databases, MIDB and IAOD, and incorporated the results of this comparison in our design process. In addition to its ease of use, the resulting product offers high flexibility in querying and downloading subsets of information that interest the user in multiple commonly used file formats. A very important design feature of MMIAD is its administration interface that allows running the prediction algorithm and adding new datasets to the database. We included this feature to ensure maintainability of the database, solving a major drawback of U12DB and SpliceRack. 
At this point, we are working on developing a probabilistic grammar inference model to be used in classifying major and minor introns. Our initial analysis shows promising results and we plan to experiment with using our grammatical solution independently or coupling it with the commonly used PWM approach. Once we have our prediction method finalized, we will use it to generate results for different species and populate the database with the resulting data. Part of our future plans is to create Galaxy (Afgan, et al., 2018) wrappers to make MMIAD accessible through the Galaxy portal.

\section{REFERENCES}

Afgan, E., Baker, D., Batut, B., van den Beek, M., Bouvier, D., Čech, M., . . . Blankenberg, D. (2018). The Galaxy platform for accessible, reproducible and collaborative biomedical analyses. Nucleic Acids Research, 46(W1), W537-W544.

Alioto, T. S. (2006). U12DB: a database of orthologous U12-type spliceosomal introns. Nucleic Acids Research, 35.

Baumgartner, M., Lemoine, C., Al Seesi, S., Karunakaran, D., Sturrock, N., Banday, A., . . Kanadia, R. (2015). Minor splicing snRNAs are enriched in the developing mouse CNS and are crucial for survival of differentiating retinal neurons. Dev Neurobiol , 75, $895-907$.

Baumgartner, M., Olthof, A., Aquino, G., Lemoine, C., Drake, K., Karunakaran, D., . . Kanadia, R. (2018). Minor spliceosome inactivation causes microcephaly, owing to cell cycle defects and death of self-amplifying radial glial cells. Development , 145(17), dev166322.

Bolchini, D., Finkelstein, A., Perrone, V., \& Nagl, S. (2009). Better bioinformatics through usability analysis. Bioinformatics, 25(3), 406-412.

D, K., AS, H., TS, F., KM, R., CW, S., D, H., \& WJ, K. (2004). The UCSC Table Browser data retrieval tool. Nucleic Acids Res., 1(32 (Database issues)), 493-6.

Holtzblatt, K., Wendell, J. B., \& Wood, S. (2004). Rapid contextual design: A how-to guide to key techniques for user-centered design. San Francisco, CA: Elsevier.

Javahery, H., Seffah, A., \& Radhakrishnan, T. (2004). Beyond power: making bioinformatics tools user centered. Comms $A C M, 47,59-62$.

Madan, V., Kanojia, D., Li, J., Okamoto, R., Sato-Otsubo, A., Kohlmann, A., . . Koeffler, H. (2015). Aberrant splicing of U12-type introns is the hallmark of ZRSR2 mutant myelodysplastic syndrome. Nat Commun, 6, 1-14.

Mao, J., Vredenburg, K., Smith, P., \& Carey, T. (2005). The state of user-centered design practice. Commun. ACM, 48(3), 105-109.

Moyer, D. C., Larue, G. E., Hershberger, C. E., Roy, S. W., \& Padgett, R. A. (2020). Comprehensive database and evolutionary dynamics of U12-type introns. Nucleic Acids Research, gkaa464.

Nielson, J. (1983). Usability engineering. New York: Morgan Kaufmann.

Niemelä, E., \& Frilander, M. (2014). Regulation of gene expression through inefficient splicing of U12-type introns. RNA Biol, 11, 1325-1329.

Norman, D. A. (2002). The psychology of everyday, 2nd edition. New York: Basic Books.

Olthof, A. M., Hyatt , K. C., \& Kanadia, R. N. (2019). Minor Intron Splicing Revisited: Identification of New Minor Intron Containing Genes and Tissue-Dependent Retention and Alternative Splicing of Minor Introns. BMC Genomics, 20(1).

Pavelin, K., Cham, J. A., de Matos, P., Brooksbank, C., \& Cameron, G. (2012). Bioinformatics Meets User-Centred Design: A Perspective. PLOS Computational. Biology, 8, 7 .

Rose, A. F. (51-60). Using qualitative studies to improve the usability of an EMR. Biomed. Inform, 38, 51-60.

Sharp, A. P., \& Burge, C. B. (1997). Classification of Introns: U2-Type or U12-Type. Cell, 91, 875-879.

Sheth, N., Roca, X., Hastings, M., Roeder, T., AR, K., \& R., S. (2006). Comprehensive Splice-Site Analysis Using Comparative Genomics. Nucleic Acids Research, 34(14), 3955-3967.

Turunen, J., Niemela, E. H., \& Verma, B. (2013). The significant other: splicing by the minor spliceosome. WIREs RNA, $4,61-76$ 